\title{
Crystal structure of mer-trans-iodomethyl-(3-methoxy-2-oxobenzoyl)- bis(trimethylphosphine)nickel(II), $\mathrm{Ni}\left[\left(\mathrm{P}_{(}\left(\mathrm{CH}_{3}\right)_{3}\right)_{2}\left(\mathrm{C}_{8} \mathrm{H}_{6} \mathrm{O}_{3}\right) \mathrm{CH}_{3}\right] \mathrm{I}$
}

\author{
Qibao Wang*, Ruifang Ding, Xiangao Quan and Daodong Zhang \\ Jining Medical University, College of Pharmaceutical Science, Rizhao 276826, P. R. China
}

Received November 1, 2008, accepted and available on-line January 22, 2008; CCDC no. 1267/2496

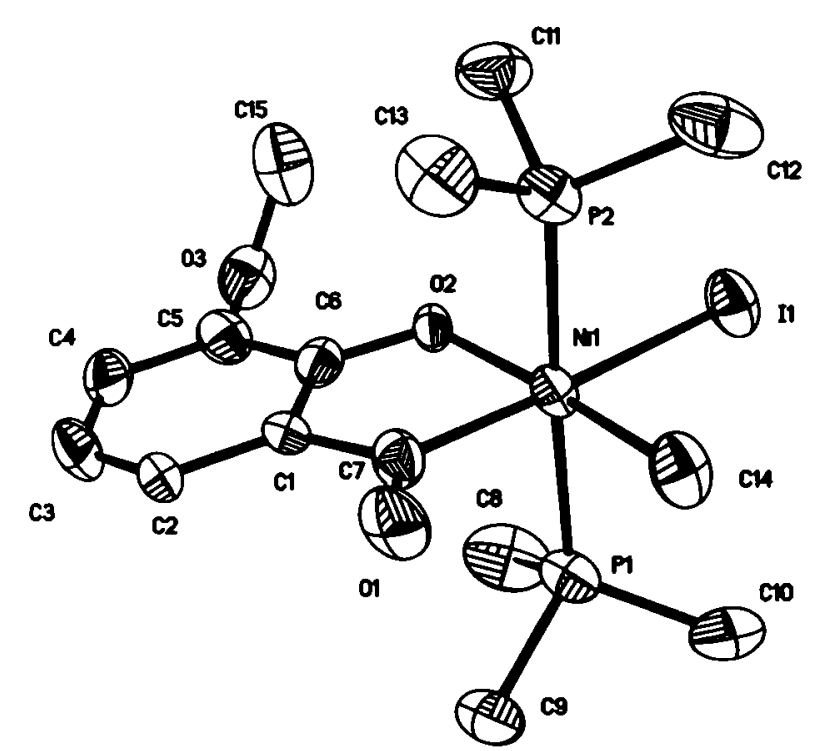

Abstract

$\mathrm{C}_{15} \mathrm{H}_{27} \mathrm{INiO}_{3} \mathrm{P}_{2}$, monoclinic, $P 121 / n 1$ (no. 14),

$a=11.3974(8) \AA, b=13.0518(7) \AA, c=13.7797(9) \AA$,

$\beta=91.323(5)^{\circ}, V=2049.3 \AA^{3}, Z=4, R_{g t}(F)=0.085$,

$w R_{\text {reff }}\left(F^{2}\right)=0.285, T=273 \mathrm{~K}$.

\section{Source of material}

Onto (3-methoxy-2-oxobenzoyl)-tris(trimethylphosphine)nickel $(1.00 \mathrm{~g}, 2.09 \mathrm{mmol})$ in $70 \mathrm{ml}$ of ether at $-70^{\circ} \mathrm{C}$ was condensed $\mathrm{CH}_{3} \mathrm{I}$ (600 mg, $4.22 \mathrm{mmol}$ ). The mixture was allowed to warm and was kept at $20^{\circ} \mathrm{C}$ for $17 \mathrm{~h}$. From the filtered solution at $-27^{\circ} \mathrm{C}$ orange-yellow crystals were obtained.

\section{Experimental details}

All $\mathbf{H}$ atoms were positioned geometrically $(d(C-H)=0.93$ $0.96 \AA)$, and refined as riding with $U_{\text {iso }}(\mathrm{H})=1.2 U_{\text {eq }}$ (carrier) or $1.5 U_{\text {eq }}$ (methyl groups). The large $R$ values may result from the air and temperature sensitivity of the title compound. The diffraction intensity was gradually weakened during the experiment.

\section{Discussion}

C,C-coupling reactions are effectively catalyzed by organophosphine-supported nickel(II) complexes [1]. In systems where neither spontaneous reduction to nickel $(0)$ nor radicals are indicated, the mechanism, which usually consists of an addition/elimination reaction sequence, requires the presence of nickel(II) intermediates [2]. Klein and his co-workers reported two types of orgamonickel(II) compounds supported by trimethylphosphine through oxidative addition reaction [3], but to date few organonickel(II) compounds have actually been investigated by $X$-ray diffraction. In this paper we reported the synthesis and the crystal structure of the title compound, which Klein had tried to synthesize [3].

In the title compound, the nickel atom is octahedrally coordinated, surrounded by two trans trimethyl-phosphine ligands and a meridional sequence of phenolato, acyl, methyl, and iodo ligands. The 3-methoxy-2-oxobenzoyl group acts as a bidentate ligand chelated to the nickel atom with the angle $86.7(4)^{\circ}$.

Table 1. Data collection and handling.

$\begin{array}{ll}\text { Crystal: } & \begin{array}{l}\text { orange-yellow block, } \\ \text { size } 0.24 \times 0.27 \times 0.35 \mathrm{~mm}\end{array} \\ \text { Wavelength: } & \text { Mo } K_{\alpha} \text { radiation }(0.71073 \AA) \\ \mu: & 26.17 \mathrm{~cm}^{-1} \\ \text { Diffractometer, scan mode: } & \text { Bruker SMART CCD, } \varphi / \omega \\ 2 \theta_{\text {max: }} & 55.12^{\circ} \\ N(h k)_{\text {measured }}, N(h k l)_{\text {miniqu: }}: & 10451,4694 \\ \text { Criterion for } I_{\text {obs }}, N(h k l)_{\text {gt }}: & I_{\text {obs }}>2 \sigma\left(I_{\text {obs }}\right), 2256 \\ N(\text { param })_{\text {refined: }} & 207 \\ \text { Programs: } & \text { SHELXS-97 [4], SHELXI-97 [5], } \\ & \text { SHELXTL [6] }\end{array}$

Table 2. Atomic coordinates and displacement parameters (in $\AA^{2}$ ).

\begin{tabular}{llllll}
\hline Atom & Site & $x$ & $y$ & $z$ & $U_{\text {iso }}$ \\
\hline H(2) & $4 e$ & 0.7674 & 0.8173 & 0.1551 & 0.081 \\
H(3) & $4 e$ & 0.8750 & 0.6757 & 0.1696 & 0.100 \\
H(4) & $4 e$ & 0.7972 & 0.5251 & 0.2168 & 0.086 \\
H(8A) & $4 e$ & 0.3385 & 0.5878 & 0.0171 & 0.163 \\
H(8B) & $4 e$ & 0.4737 & 0.6088 & 0.0116 & 0.163 \\
H(8C) & $4 e$ & 0.3939 & 0.6170 & -0.0823 & 0.163 \\
H(9A) & $4 e$ & 0.4683 & 0.8115 & -0.1278 & 0.127 \\
H(9B) & $4 e$ & 0.5596 & 0.7996 & -0.0417 & 0.127 \\
H(9C) & $4 e$ & 0.4801 & 0.8975 & -0.0488 & 0.127 \\
H(10A) & $4 e$ & 0.2446 & 0.7671 & -0.1206 & 0.174 \\
H(10B) & $4 e$ & 0.2329 & 0.8674 & -0.0590 & 0.174 \\
H(10C) & $4 e$ & 0.1740 & 0.7656 & -0.0243 & 0.174 \\
H(11A) & $4 e$ & 0.4333 & 0.6785 & 0.3952 & 0.130 \\
H(11B) & $4 e$ & 0.2958 & 0.6744 & 0.3906 & 0.130 \\
H(11C) & $4 e$ & 0.3605 & 0.7367 & 0.4731 & 0.130 \\
H(12A) & $4 e$ & 0.1613 & 0.8578 & 0.3390 & 0.140 \\
H(12B) & $4 e$ & 0.2304 & 0.9613 & 0.3491 & 0.140 \\
H(12C) & $4 e$ & 0.2231 & 0.8869 & 0.4381 & 0.140 \\
H(13A) & $4 e$ & 0.4653 & 0.9122 & 0.4505 & 0.132 \\
H(13B) & $4 e$ & 0.4672 & 0.9749 & 0.3535 & 0.132 \\
H(13C) & $4 e$ & 0.5486 & 0.8789 & 0.3671 & 0.132 \\
H(14A) & $4 e$ & 0.3325 & 0.9766 & 0.1897 & 0.105 \\
H(14B) & $4 e$ & 0.2260 & 0.9281 & 0.1321 & 0.105 \\
& & & & & \\
\hline & & & & &
\end{tabular}

* Correspondence author (e-mail: qibaowang@sina.com) 
Table 2. Continued.

\begin{tabular}{llllll}
\hline Atom & Site & $x$ & $y$ & $z$ & $U_{\text {iso }}$ \\
\hline H(14C) & 4e & 0.3431 & 0.9518 & 0.0789 & 0.105 \\
H(15A) & 4e & 0.5797 & 0.4748 & 0.3734 & 0.148 \\
\hline
\end{tabular}

Table 2. Continued.

\begin{tabular}{llllll}
\hline Atom & Site & $x$ & $y$ & $z$ & $U_{\text {iso }}$ \\
\hline H(15B) & $4 e$ & 0.4900 & 0.4009 & 0.3213 & 0.148 \\
H(15C) & $4 e$ & 0.4654 & 0.5190 & 0.3237 & 0.148
\end{tabular}

Table 3. Atomic coordinates and displacement parameters (in $\AA^{2}$ ).

\begin{tabular}{|c|c|c|c|c|c|c|c|c|c|c|}
\hline Atom & Site & $x$ & $y$ & $z$ & $U_{11}$ & $U_{22}$ & $U_{33}$ & $U_{12}$ & $U_{13}$ & $U_{23}$ \\
\hline $\mathbf{N i}(1)$ & $4 e$ & $0.3698(1)$ & $0.78862(9)$ & $0.1722(1)$ & $0.0373(7)$ & $0.0412(7)$ & $0.0584(8)$ & $0.0005(5)$ & $0.0108(6)$ & $0.0001(6)$ \\
\hline$O(1)$ & $4 e$ & $0.5588(7)$ & $0.9260(6)$ & $0.1505(7)$ & $0.063(5)$ & $0.064(5)$ & $0.106(7)$ & $-0.026(4)$ & $0.014(5)$ & $0.009(5)$ \\
\hline$O(2)$ & $4 e$ & $0.4404(5)$ & $0.6510(5)$ & $0.2029(4)$ & $0.030(3)$ & $0.049(4)$ & $0.052(4)$ & $0.006(3)$ & $0.002(3)$ & $0.010(3)$ \\
\hline$O(3)$ & $4 e$ & $0.5839(8)$ & $0.4793(7)$ & $0.2341(7)$ & $0.080(6)$ & $0.069(6)$ & $0.084(6)$ & $0.024(5)$ & $-0.005(5)$ & $-0.016(5)$ \\
\hline $\mathbf{P}(1)$ & $4 e$ & $0.3727(3)$ & $0.7613(3)$ & $0.0114(2)$ & $0.061(2)$ & $0.067(2)$ & $0.057(2)$ & $-0.013(2)$ & $0.014(1)$ & $-0.006(2)$ \\
\hline$P(2)$ & $4 e$ & $0.3600(3)$ & $0.8277(2)$ & $0.3313(2)$ & $0.064(2)$ & $0.055(2)$ & $0.064(2)$ & $-0.001(1)$ & $0.015(2)$ & $-0.002(1)$ \\
\hline I(1) & $4 e$ & $0.16002(7)$ & $0.69392(6)$ & $0.17812(7)$ & $0.0494(5)$ & $0.0614(6)$ & $0.1083(8)$ & $-0.0045(3)$ & $0.0167(4)$ & $0.0028(4)$ \\
\hline$C(1)$ & $4 e$ & $0.6104(9)$ & $0.750(1)$ & $0.1775(7)$ & $0.040(6)$ & $0.083(8)$ & $0.046(6)$ & $0.001(6)$ & $0.009(4)$ & $0.024(6)$ \\
\hline$C(2)$ & $4 e$ & $0.7314(9)$ & $0.755(1)$ & $0.1688(8)$ & $0.034(6)$ & $0.11(1)$ & $0.059(7)$ & $0.000(6)$ & $0.007(5)$ & $0.028(7)$ \\
\hline$C(3)$ & $4 e$ & $0.795(1)$ & $0.672(1)$ & $0.180(1)$ & $0.041(7)$ & $0.13(1)$ & $0.077(9)$ & $0.016(8)$ & $0.022(6)$ & $-0.006(9)$ \\
\hline$C(4)$ & $4 e$ & $0.749(1)$ & $0.582(1)$ & $0.2051(9)$ & $0.053(7)$ & $0.10(1)$ & $0.065(8)$ & $0.028(7)$ & $0.004(6)$ & $0.001(7)$ \\
\hline$C(5)$ & $4 e$ & $0.629(1)$ & $0.5743(9)$ & $0.2136(8)$ & $0.062(7)$ & $0.059(7)$ & $0.060(7)$ & $0.004(6)$ & $0.011(6)$ & $-0.012(6)$ \\
\hline$C(6)$ & $4 e$ & $0.5545(9)$ & $0.6558(8)$ & $0.1979(7)$ & $0.052(6)$ & $0.057(6)$ & $0.039(5)$ & $0.014(5)$ & $-0.003(5)$ & $-0.010(5)$ \\
\hline$C(7)$ & $4 e$ & $0.5303(9)$ & $0.8398(9)$ & $0.1665(8)$ & $0.041(6)$ & $0.063(7)$ & $0.059(7)$ & $-0.007(5)$ & $-0.001(5)$ & $-0.005(5)$ \\
\hline$c(10)$ & $4 e$ & $0.241(2)$ & $0.794(1)$ & $-0.056(1)$ & $0.11(1)$ & $0.17(2)$ & $0.068(9)$ & $0.01(1)$ & $0.006(9)$ & $0.04(1)$ \\
\hline C(11) & $4 e$ & $0.363(2)$ & $0.717(1)$ & $0.406(1)$ & $0.12(1)$ & $0.078(9)$ & $0.066(8)$ & $0.024(8)$ & $0.018(8)$ & $0.015(7)$ \\
\hline$C(12)$ & $4 e$ & $0.228(1)$ & $0.891(1)$ & $0.369(1)$ & $0.14(1)$ & $0.061(8)$ & $0.080(9)$ & $0.006(8)$ & $0.045(9)$ & $-0.009(6)$ \\
\hline$C(13)$ & $4 e$ & $0.473(1)$ & $0.908(1)$ & $0.381(1)$ & $0.09(1)$ & $0.081(9)$ & $0.10(1)$ & $-0.022(8)$ & $0.007(8)$ & $-0.023(8)$ \\
\hline$C(14)$ & $4 e$ & $0.310(1)$ & $0.9297(8)$ & $0.139(1)$ & $0.062(7)$ & $0.046(6)$ & $0.102(9)$ & $0.005(5)$ & $0.011(7)$ & $-0.009(6)$ \\
\hline$C(15)$ & $4 e$ & $0.525(1)$ & $0.468(1)$ & $0.320(1)$ & $0.07(1)$ & $0.051(7)$ & $0.17(2)$ & $-0.010(7)$ & $0.01(1)$ & $0.005(9)$ \\
\hline
\end{tabular}

\section{References}

1. Parshall, G.; Ittel, S. D.: Homogeneous Catalysis. Chapter 7.4. 2nd ed. Wiley, New York, 1992.

2. Klein, H. F.; Bickelhaupt, A.; Jung, T.; Cordier, G.: Syntheses and Properties of the First Octahedral Diorganonickel(IV) Compounds. Organometallics 13 (1994) 2557-2559.

3. Klein, H. F.; Bickelhaupt, A.; Lemke, M.; Sun, H. J.; Brand, A.; Jung, T.; Röhr, C.; Flörke, U.; Haupt, H.: Trimethylphosphine Complexes of Diorganonickel(IV) Moieties. J. Organometallics 16 (1997) 668-676.
4. Sheldrick, G. M.: SHELXS-97. Program for the Solution of Crystal Structures. University of Göttingen, Germany 1997.

5. Sheldrick, G. M.: SHELXI-97. Program for the Refinement of Crystal Structures. University of Göttingen, Germany 1997.

6. Sheldrick, G. M.: SHELXTL. Structure Determination Software Suite. Version 6.14. Bruker AXS, Madison, Wisconsin, USA 2000 